\title{
The Effect of Laser Acupuncture on Semen Parameters in Infertile Men With Oligospermia: A Randomized Clinical Trial
}

\author{
Farzad Allameh $^{1}{ }^{\circledR}$, Mohammadreza Razzaghi', Sedighe Hosseini², Maryam Barati' ${ }^{1}$, Zahra Razzaghi ${ }^{3}$, \\ Safa Salehi ${ }^{4}$, Seyed Mohammad Ghahestani ${ }^{5}$, Vahid Shahabi ${ }^{*}(\mathbb{D})$ \\ ${ }^{1}$ Laser Application in Medical Sciences Research Center, Shahid Beheshti University of Medical Sciences, Tehran, Iran \\ ${ }^{2}$ Department of Infertility \& IVF, Taleghani Hospital, Shahid Beheshti University of Medical Sciences, Tehran, Iran \\ ${ }^{3}$ Center of Excellence in Training Laser Application in Medicine, Shahid Beheshti University of Medical Sciences, \\ Tehran, Iran \\ ${ }^{4}$ Department of Gynecology and Obstetrics, School of Medicine, Shahid Beheshti University of Medical Sciences, \\ Tehran, Iran \\ ${ }^{5}$ Pediatric Urology Ward, Children Medical Center Hospital, Tehran University of Medical Sciences \\ ${ }^{6}$ Department of Urology, School of Medicine, Shahid Beheshti University of Medical Sciences, Tehran, Iran
}

\section{*Correspondence to Vahid Shahabi, School of Medicine, Shahid Beheshti University of Medical Sciences, Koudakyar Ave., Daneshjoo Blv. Velenjak, Tehran, Iran. Email: khafash88@gmail.com}

Received: September 2, 2021 Accepted: November 16, 2021 Published online 27 December 2021

\begin{abstract}
Introduction: Currently, infertility is a main concern, and it is estimated that $15 \%$ of couples are considered infertile. Conventional therapies such as vitamins, antioxidants and supplements have been used widely, but they may not be sufficiently effective. This study evaluated the effect of laser acupuncture on semen parameters in infertile patients with oligospermia.

Methods: This study is a clinical trial with a double-blind control group. 35 patients in the intervention group underwent laser acupuncture twice a week for consecutive 5-weeks. In the control group, sham laser acupuncture was performed. The two groups were compared in terms of semen parameters including semen volume, sperm concentration, sperm motility and sperm morphology as the primary outcome. The secondary outcome was a successful pregnancy rate. After data collection, statistical analysis was performed with SPSS version 20.

Results: The mean age of all patients was $27.46 \pm 2.88$ years; in the intervention group, their age was $27.82 \pm 2.6$ years, and in the control group, it was $27.11 \pm 2.96$ years, and there was no significant difference $(P=0.396)$. During the study, 4 patients $(5.4 \%)$ reported adverse effects; three patients in the control group complained of mild weakness and itching, and one patient in the intervention group reported dizziness. The volume of semen and sperm morphology did not differ significantly in both control and intervention groups $(P>0.05)$, while sperm motility and sperm concentration had a significant difference $(P=0.0001)$.

Conclusion: Laser acupuncture as a non-invasive procedure is effective in improving sperm mobility and concentration in infertile patients with oligospermia. Due to the surface contact of the laser probe with the skin and the lack of need to insert needles in the skin, laser acupuncture is more convenient and better tolerated by patients.

Keywords: Acupuncture, Infertility, Laser, Semen analysis, Spermatozoa
\end{abstract}

\section{Introduction}

Infertility is defined as the failure to achieve pregnancy after 12 months of unprotected coitus. It is estimated that $15 \%$ of couples are considered infertile. Approximately $35 \%$ are only due to female factors, $30 \%$ are only due to male factors, $20 \%$ are due to a combination of male and female factors, and 15\% remain unexplained. ${ }^{1}$ However, male diseases that affect fertility are still common. It is not diagnosed or treated. ${ }^{1,2}$

Male infertility patterns are very different in different regions. The highest fertility rate is reported in Finland, while the United Kingdom has the lowest fertility rate. It seems that a combination of social habits, environmental conditions and genetics play a role in this difference. ${ }^{3}$ In Iran, according to studies, the overall prevalence of infertility is reported to be between $8 \%$ and $25 \% .{ }^{4}$

Conventional therapies such as over-the-counter vitamins, antioxidants and supplements have been used widely, but they may not be effective in improving semen parameters and the true effects on dosing regimens could not be identified. ${ }^{5,6}$ Other studies have demonstrated that acupuncture treatment improves sperm parameters of men suffering from impaired sperm quality. ${ }^{7-11}$

Acupuncture is a medical technique unique to 
traditional Chinese medicine and has been practiced for more than 2000 years to treat a whole range of illnesses. It performs via inserting needles at various points along the body that are called acupoints. ${ }^{12}$ Acupuncture is safe, but it also has risks. The common adverse effects of acupuncture have been needle pain $(1 \%-45 \%)$ from treatments, tiredness $(2 \%-41 \%)$, and bleeding $(0.03 \%-$ $38 \%)$. Several large prospective studies have also shown that these mild, transient adverse effects occur in about $7 \%$ to $11 \%$ of all cases. ${ }^{13-15}$

To avoid the adverse effects of conventional acupuncture, a low-level laser has been used in several clinical trials in many fields including orthopedics, pain attenuation, internal medicine, sports medicine, rheumatology, and dermatology. ${ }^{15}$ In the current study, it is aimed to evaluate the effect of laser acupuncture treatment on semen parameters in infertile men with oligospermia, who live in Iran.

\section{Materials and Methods}

The study was approved by the ethics committee of Shahid Beheshti University of Medical Sciences (IR.SBMU.SRC. REC.1399.005), and informed consent was obtained from all patients according to the hospital's guidelines. The registration code in the Iranian Registry of Clinical Trial is IRCT20200624047907N1. Upon previous studies, ${ }^{8,9}$ the sample size was determined by using the following formula:

$n=\frac{\left(Z_{1-\alpha / 2}+Z_{1-\beta}\right)^{2}\left(S D_{1}^{2}+S D_{2}^{2}\right)}{d^{2}}$

Infertile men under 35 years of age referred to the urology clinic of Shohada-e-Tajrish and Taleghani Hospital were evaluated for eligibility to enter the study.

\section{Inclusion Criteria}

Men were under 35 years of age, with at least one year of unsuccessful attempt for conception, who had oligospermia (sperm concentration less than 15 million per milliliter) and primary infertility.

Exclusion criteria included azoospermia, a history of infertility-related surgery (varicocele, undescended testis, and inguinal hernia), a history of hormonal and genetic disorders, a history of hormone replacement therapy in recent 3 years, presence of varicocele on examination or Ultrasound, a history of undescended testis, a history of mumps and epididymo-orchitis, a history of medical diseases (diabetes, hypertension, hyperlipidemia, thyroid disorders, etc.), abnormal examination of the testicles, a history of smoking, alcohol and drug use, as well as a history of occupational exposure with chemicals and radioactive agents.

Eligible patients randomly entered the two parallel groups, the intervention group or the control group, and this process continued until the sample size was completed (35 patients for each group). The consort flow diagram is shown in Figure 1. The random allocation sequence was generated using a computer-based random number generator to select random permuted blocks with six patients. Sealed numbered containers were used to implement the random allocation sequence. The sequence was concealed until interventions were assigned. The code was revealed for statistical evaluation once recruitment and data collection were complete. Generation of the allocation sequence, enrollment, and assignment of patients were carried out separately. All patients and study personnel were blinded to group assignment for the duration of the study. Only the laser acupuncture specialists knew the type of intervention but were blinded to laboratory data.

The intervention was in the form of transfer of light energy by laser fibers to several specific points, which was performed by trained technicians of acupuncture and lasers, using an 810-nm laser with a power of $300 \mathrm{~mW}$ and continuously with the energy of 18 joules per minute. It is irradiated at 90 degrees on the skin for a period of 5 to 10 seconds and the 21 points of irradiation were as follows: HN1, LI4, ST36, GB34, SP9, SP6, LR3, RN12, RN6, RN4, RN2, ST29, ST25, GB20, DU14, BL18, BL19, BL20, BL21, BL25, and BL32.

Patients in the intervention group underwent laser acupuncture twice a week for consecutive 5-weeks. In the control group, sham laser acupuncture was performed. In case of any complication, it was recorded in the patient's file.

Three semen samples were taken from all patients. The first sample was taken before the intervention, the second sample was taken immediately after the intervention was completed, and the third sample was taken three months after the completion of the intervention. It should be noted that all samples were analyzed in the andrology laboratory, Infertility and IVF Center, Taleghani Hospital. The two groups were compared in terms of semen parameters including semen volume, sperm concentration, sperm motility and sperm morphology as the primary outcome. The secondary outcome was a successful pregnancy rate.

The Mann-Whitney $U$ test was applied to compare continuous parameters. The Wilcoxon matched-pairs test was used for the comparison of dependent variables before versus after the intervention. Categorical variables are presented as $\mathrm{n}(\%)$. Continuous variables are presented as mean \pm standard deviation (SD). All data were analyzed through SPSS Statistics, version 20.0 (IBM Corp., Armonk, NY, USA). The significance level was set at 0.05.

\section{Results}

In this study, 70 infertile men with oligospermia were studied. The mean age of all patients was $27.46 \pm 2.88$ years; in the intervention group, it was $27.82 \pm 2.6$ years and in the control group, it was $27.11 \pm 2.96$ years, and there 


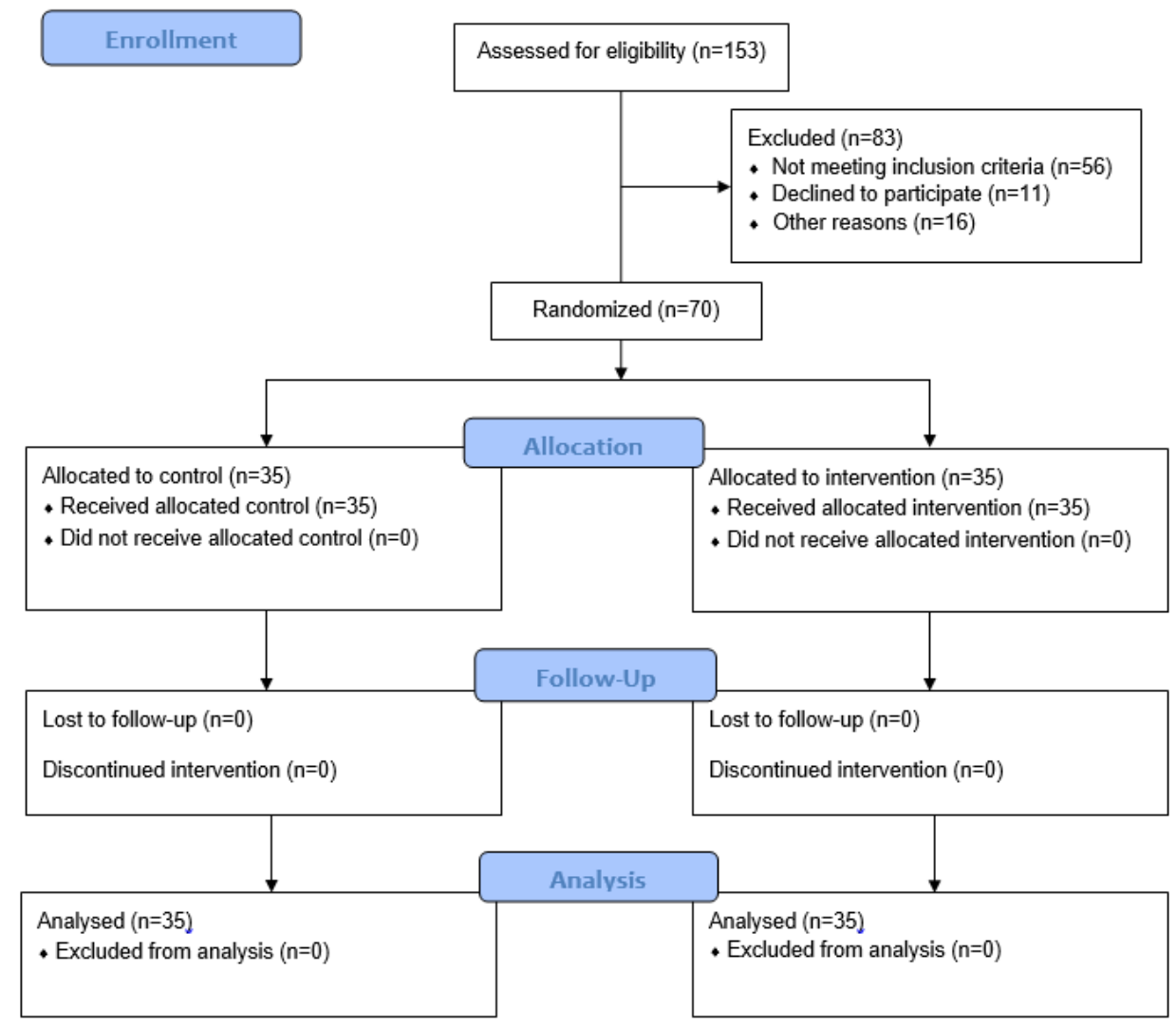

Figure 1. Consort Flow Diagram of the Study.

was no significant difference $(P=0.396)$. Demographic characteristics are shown in Table 1.

During the study, 4 patients (5.4\%) reported adverse effects; three patients in the control group reported mild weakness and itching, and one patient in the intervention group complained of dizziness. Each of these adverse effects occurred only once and was resolved in less than 24 hours without any treatments.

Three semen samples were taken from all patients. The first sample was taken before the intervention, the second sample was taken immediately after the intervention was completed, and the third sample was taken three months after the completion of the intervention. The results of semen analysis are given in Tables 2-5 and Figures 2-5. Because of short-term follow-up, no successful conception was observed in any group.

\section{Discussion}

The present study was a clinical trial, and the effect of laser acupuncture on the infertility of 70 men with oligospermia was investigated. The first semen analysis was performed before the onset of the intervention, the second semen analysis was performed immediately after the end of the intervention, and the third semen analysis was performed 3 months after the end of the intervention. Due to the length of the spermatogenesis period (nearly 2 months), data analysis was conducted by comparing the first and second semen samples with the third sample. The volume of semen and sperm morphology did not differ significantly in both control and intervention groups $(P>$ $0.05)$, while sperm motility and sperm concentration had a significant difference $(P=0.0001)$.

In the majority of studies and clinical trials, the sample size was less than $50,^{7,8,10-18}$ and intervention was performed in the needle acupuncture method. ${ }^{7,8,10,15}$ In Siterman's study, ${ }^{8} 40$ patients participated in both intervention and control groups. At the end of the study, the effect of acupuncture on sperm concentration was significant. The main objection to that study was the lack of sham acupuncture in the control group, which caused bias by the procedure. However, the results of that study are consistent with the current study.

In another study, ${ }^{17}$ the sample size was approximately similar to the current study (each group 29 patients), and also a similar acupuncture effect on sperm motility was noted. A significant decrease in semen fluid volume after acupuncture $(P=0.041)$ was seen, which was against the current study. According to the author of that study, a decrease in semen fluid volume was probably due to the small sample size; if the sample size was above 45 patients in each group, the observed difference was significant in semen volume.

In a follow-up study, ${ }^{11}$ contrary to the current study, the reduction of sperm morphology defects after acupuncture was reported. Twelve infertile patients were subjected to acupuncture for 5 weeks and after 2 months of sperm 
morphology with electron microscopy. Considering that in the current study, sperm morphology was evaluated by an optical microscope, the probable cause of the difference in the results of the two studies can be due to the difference in the use of an optical microscope versus

Table 1. Demographic characteristic

\begin{tabular}{lcccc}
\hline & Control Group & Intervention Group & Total & $\boldsymbol{P}$ Value \\
\hline Cases $(\mathrm{n})$ & 35 & 35 & 70 & \\
& & & & \\
$\begin{array}{l}\text { Age (mean } \\
\text { years } \pm \text { SD) }\end{array}$ & $27.11 \pm 2.96$ & $27.82 \pm 2.6$ & $27.46 \pm 2.88$ & 0.396 \\
& & & &
\end{tabular}

Table 2. The Effect of Acupuncture Treatment on Mean Semen Volume

\begin{tabular}{cccc}
\hline Semen Analysis & Intervention Group & Control Group & $\boldsymbol{P}$ Value \\
\hline $1^{\text {st }}$ Sample & $3.44 \pm 0.44$ & $3.44 \pm 0.4$ & 0.746 \\
$2^{\text {nd }}$ Sample & $3.42 \pm 0.41$ & $3.44 \pm 0.42$ & 0.944 \\
$3^{\text {rd }}$ Sample & $3.50 \pm 0.43$ & $3.40 \pm 0.39$ & 0.150 \\
\hline
\end{tabular}

Table 3. The Effect of Acupuncture Treatment on Mean \% Sperm Motility (A and $\mathrm{B}$ )

\begin{tabular}{cccc}
\hline Semen Analysis & Intervention Group & Control Group & $\boldsymbol{P}$ Value \\
\hline $1^{\text {st }}$ Sample & $27.77 \pm 13.00$ & $27.06 \pm 14.17$ & 0.746 \\
$2^{\text {nd }}$ Sample & $28.45 \pm 12.71$ & $27.77 \pm 13.98$ & 0.672 \\
$3^{\text {rd }}$ Sample & $44.48 \pm 14.3$ & $27.97 \pm 14.29$ & 0.0001
\end{tabular}

Table 4. The Effect of Acupuncture Treatment on Mean Sperm Concentration (million/mL)

\begin{tabular}{cccc}
\hline Semen Analysis & Intervention Group & Control Group & $\boldsymbol{P}$ Value \\
\hline $1^{\text {st }}$ Sample & $13.14 \pm 1.21$ & $13.22 \pm 1.21$ & 0.690 \\
$2^{\text {nd }}$ Sample & $13.40 \pm 1.61$ & $13.28 \pm 1.72$ & 0.933 \\
$3^{\text {rd }}$ Sample & $28.25 \pm 6.44$ & $13.48 \pm 1.42$ & 0.0001 \\
\hline
\end{tabular}

Table 5. The Effect of Acupuncture Treatment on Sperm Morphology (\%)

\begin{tabular}{cccc}
\hline Semen Analysis & Intervention Group & Control Group & $\boldsymbol{P}$ Value \\
\hline $1^{\text {st }}$ Sample & $1.44 \pm 0.39$ & $1.52 \pm 0.44$ & 0.397 \\
$2^{\text {nd }}$ Sample & $1.42 \pm 0.40$ & $1.51 \pm 0.41$ & 0.379 \\
$3^{\text {rd }}$ Sample & $1.45 \pm 0.39$ & $1.52 \pm 0.46$ & 0.542 \\
\hline
\end{tabular}

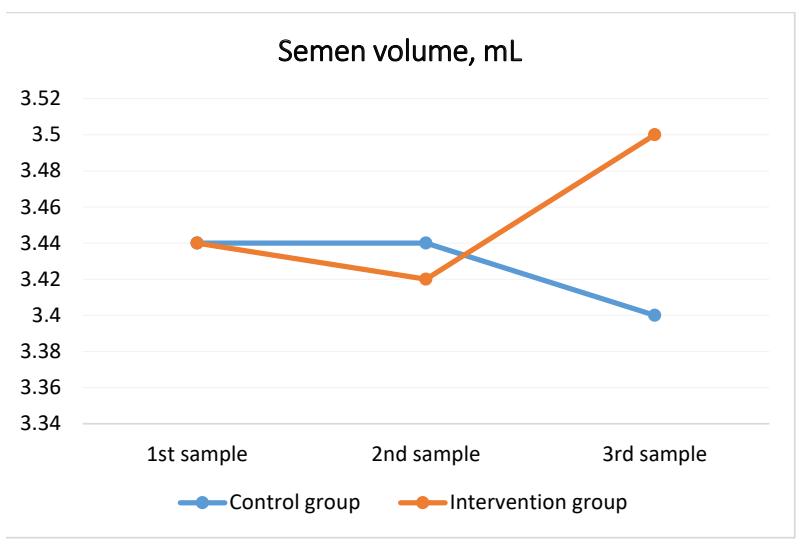

Figure 2. Comparison of the Effect of Acupuncture on Mean Semen Volume. an electron microscope. Therefore, it is recommended that in subsequent studies, sperm morphology should be evaluated by an electron microscope which has higher accuracy in identifying ultrastructure defects.

In a prospective, randomized, controlled, single-

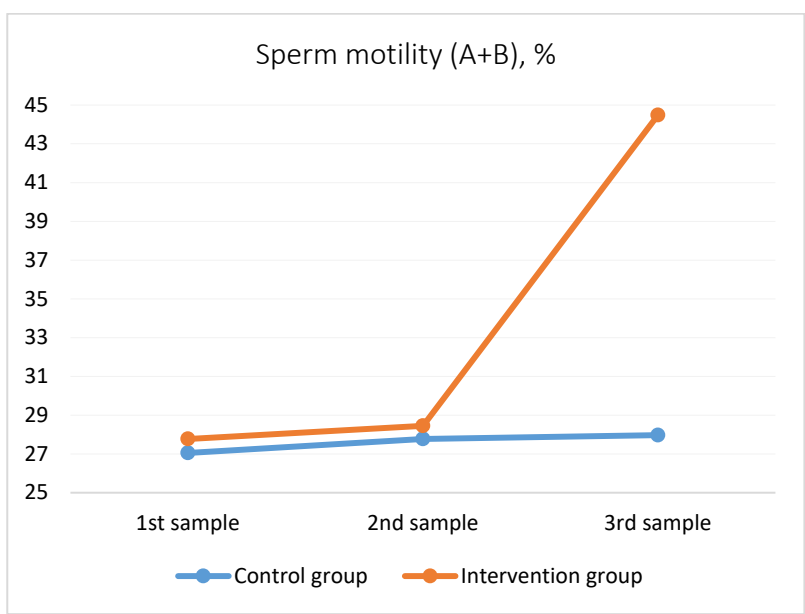

Figure 3. Comparison of the Effect of Acupuncture on Mean \% Sperm Motility $(A+B)$.

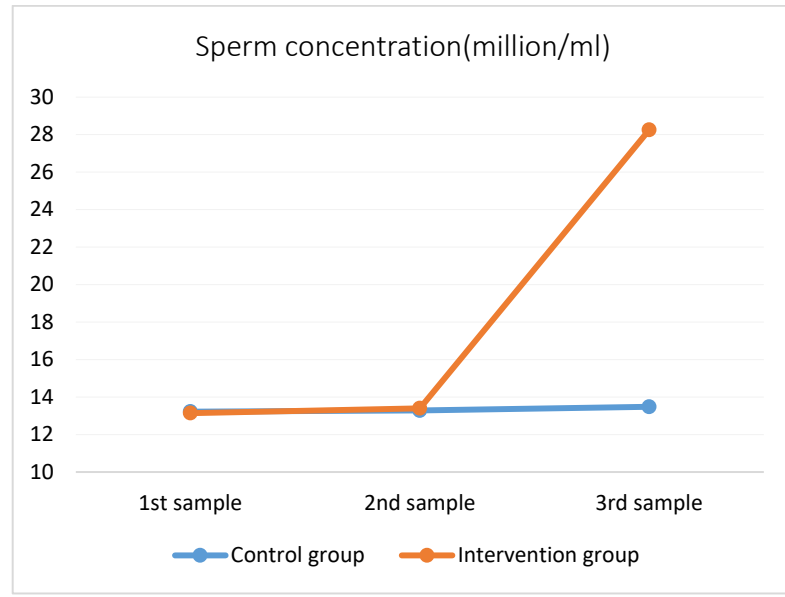

Figure 4. Comparison of the Effect of Acupuncture on Mean Sperm Concentration (million $/ \mathrm{mL}$ )

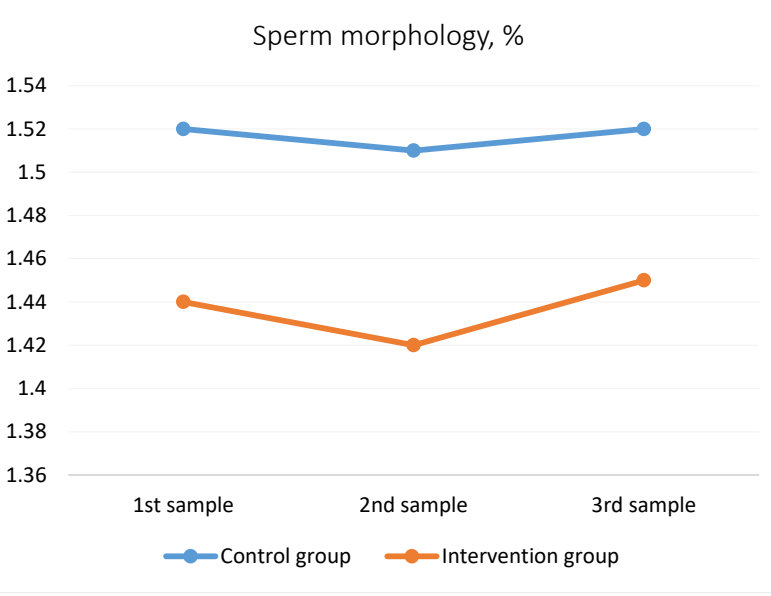

Figure 5. Comparison of the Effect of Acupuncture on Sperm Morphology (\%). 
blind study by Gurfinkel et $\mathrm{al}^{18,19}$ patients with semen abnormalities in concentration, morphology, and/or progressive motility received acupuncture and moxa treatment at therapeutic or indifferent points for 10 weeks. The percentage of morphologically normal sperm increased significantly after acupuncture and moxa treatment at therapeutic points compared with indifferent points.

\section{Conclusion}

Regarding the observations and the results of the current study, it can be concluded that laser acupuncture as a non-invasive procedure is effective in improving sperm motility and concentration in infertile patients with oligospermia. Due to the surface contact of the laser probe with the skin and the lack of need to insert needles in the skin, laser acupuncture is more convenient and better tolerated by patients.

\section{Limitations and Suggestions}

As previously mentioned, one of the limitations of this study was the lack of access to an electron microscope to evaluate sperm morphology. Another important limitation is the short-term follow-up.

Even though the majority of similar studies have had less sample size, it is recommended to conduct a trial with a large sample size in future. Also, we suggest, in subsequent studies, comparisons between the number of sessions and doses of laser energy, as well as comparisons between laser acupuncture and needle acupuncture.

\section{Authors' Contributions}

FA Suggested the idea, MR led the project, SH referred patients, ZR analyzed data, SS collected the data and did the scientific writing, and VS performed the intervention.

\section{Conflict of Interest}

None.

\section{Ethical Considerations}

The study was approved by the ethics committee of Shahid Beheshti University of Medical Sciences (IR.SBMU.SRC. REC.1399.005).

\section{Acknowledgement}

We should acknowledge the andrology laboratory personnel and expert embryologists in Taleghani Infertility Center.

\section{References}

1. Mascarenhas MN, Flaxman SR, Boerma T, Vanderpoel S, Stevens GA. National, regional, and global trends in infertility prevalence since 1990: a systematic analysis of 277 health surveys. PLoS Med. 2012;9(12): e1001356. doi: 10.1371/ journal.pmed.1001356.

2. Schlegel PN, Sigman M, Collura B, De Jonge CJ, Eisenberg ML, Lamb DJ, et al. Diagnosis and Treatment of Infertility in Men: AUA/ASRM Guideline Part I. J Urol. 2021 ;205(1):36-43. doi: 10.1097/JU.0000000000001521.

3. Yao DF, Mills JN. Male infertility: lifestyle factors and holistic, complementary, and alternative therapies. Asian J Androl. 2016;18(3):410-8. doi: 10.4103/1008-682X.175779.

4. Safarinejad MR. Infertility among couples in a populationbased study in Iran: prevalence and associated risk factors. Int $J$ Androl. 2008;31(3):303-14. doi: 10.1111/j.13652605.2007.00764. x.

5. Ko EY, Sabanegh ES Jr. The role of over-the-counter supplements for the treatment of male infertility--fact or fiction? J Androl. 2012 ;33(3):292-308. doi: 10.2164/ jandrol.111.013730.

6. Nazari L, Salehpour S, Hosseini S, Allameh F, Jahanmardi F, Azizi E, et al. Effect of antioxidant supplementation containing L-carnitine on semen parameters: a prospective interventional study. JBRA Assist Reprod. 2021;25(1):76-80. doi: 10.5935/1518-0557.20200043.

7. Ng EH, So WS, Gao J, Wong YY, Ho PC. The role of acupuncture in the management of subfertility. Fertil Steril. 2008 ;90(1):1-13. doi: 10.1016/j.fertnstert.2008.02.094.

8. Siterman S, Eltes F, Wolfson V, Lederman H, Bartoov B. Does acupuncture treatment affect sperm density in males with very low sperm count? A pilot study. Andrologia. 2000;32(1):31-9.

9. Siterman S, Eltes F, Schechter L, Maimon Y, Lederman H, Bartoov B. Success of acupuncture treatment in patients with initially low sperm output is associated with a decrease in scrotal skin temperature. Asian J Androl. 2009;11(2):200-8. doi: 10.1038/aja.2008.4.

10. Tempest HG, Homa ST, Zhai XP, Griffin DK. Significant reduction of sperm disomy in six men: effect of traditional Chinese medicine? Asian J Androl. 2005;7(4):419-25. doi: 10.1111/j.1745-7262.2005.00068.x.

11. Pei J, Strehler E, Noss U, Abt M, Piomboni P, Baccetti B, et al. Quantitative evaluation of spermatozoa ultrastructure after acupuncture treatment for idiopathic male infertility. Fertil Steril. 2005;84(1):141-7. doi: 10.1016/j.fertnstert.2004.12.056.

12. Jo J, Lee SH, Lee JM, Jerng UM. Semen Quality Improvement in a Man with Idiopathic Infertility Treated with Traditional Korean Medicine: A Case Report. Explore (NY). 2015;11(4):320-3. doi: 10.1016/j.explore.2015.04.007.

13. Ernst E, White AR. Prospective studies of the safety of acupuncture: a systematic review. Am J Med. 2001;110(6):4815. doi: 10.1016/s0002-9343(01)00651-9.

14. Fernandes GH, de Carvalho Pde T, Serra AJ, Crespilho AM, Peron JP, Rossato C, et al. The effect of low-level laser irradiation on sperm motility, and integrity of the plasma membrane and acrosome in cryopreserved bovine sperm. PLoS One. 2015;10(3): e0121487. doi: 10.1371/journal. pone.0121487.

15. Moskvin SV, Apolikhin OI. Effectiveness of low level laser therapy for treating male infertility. Biomedicine (Taipei). 2018;8(2):7. doi: 10.1051/bmdcn/2018080207.

16. Hasan P, Rijadi S, Purnomo S. The Possible Application of low Reactive-Level Laser Therapy (LLLT) in the Treatment of Male Infertility: A preliminary report. Laser therapy. 2004;14(2):6566. doi:10.5978/islsm.14.0_65.

17. Dieterle S, Li C, Greb R, Bartzsch F, Hatzmann W, Huang D. A prospective randomized placebo-controlled study of the effect of acupuncture in infertile patients with severe oligoasthenozoospermia. Fertil Steril. 2009;92(4):1340-1343. doi: 10.1016/j.fertnstert.2009.02.041.

18. Gurfinkel E, Cedenho AP, Yamamura Y, Srougi M. Effects of acupuncture and moxa treatment in patients with semen abnormalities. Asian J Androl. 2003;5(4):345-8. 